H. UEDA

KODAI MATH. J.

5 (1982), 355-359

\title{
ON THE GROWTH OF SUBHARMONIC FUNCTIONS
}

\author{
By Hideharu UEDA
}

1. Introduction. Let $u(z)$ be a subharmonic function in the complex plane $C$. We denote the order and lower order of $u(z)$ by $\rho$ and $\mu$, respectively. Let $M(r, u)$ and $m^{*}(r, u)$ denote the maximum and infimum of $u(z)$ on $|z|=r$, respectively. The classical $\cos \pi \rho$ theorem asserts that, given $\varepsilon>0$, the inequality

$$
m^{*}(r, u)>(\cos \pi \rho-\varepsilon) M(r, u)
$$

holds for a sequence $r=r_{n} \rightarrow \infty$, provided that $\rho<1$. Kjellberg [3] proved a striking improvement of this theorem.

THEOREM A. If $\lambda \in(0,1)$, then

$$
m^{*}(r, u)>\cos \pi \lambda \cdot M(r, u)
$$

on an unbounded sequence of $r$, unless

$$
r^{-1} M(r, u) \longrightarrow \alpha \quad(r \rightarrow \infty)
$$

where $\alpha$ is postive or $\infty$.

An important consequence of Theorem $\mathrm{A}$ is that if $\mu<1$ then the inequality (1) holds with $\rho$ replaced by $\mu$ on an unbounded sequence of $r$. Another important consequence of Theorem $A$ is the following fact.

"If $u(z)$ is subharmonic of order $\rho(0<\rho<1)$ and minimal type, then

$$
m^{*}(r, u)>\cos \pi \rho M(r, u)
$$

on a sequence of $r \rightarrow \infty$."

Such a fact does not always hold for subharmonic functions of order $\rho$ and mean type. Barry [2] proved the following result.

Theorem B. Let $h(r)$ be positive and continuous for $r \geqq r_{0}$, and for each $s>0$,

$$
\frac{h(s r)}{h(r)} \longrightarrow 1 \quad(r \rightarrow \infty) .
$$

Suppose that $h(r) \rightarrow 0 \quad(r \rightarrow \infty)$ and $h^{\prime}(r)>-O\left(r^{-1}\right)(r \rightarrow \infty)$. If $u(z)$ is subharmonc of order $\rho(0<\rho<1 / 2)$ and mean type, and

Received February 9, 1981 


$$
\int^{\infty} h(t) \frac{d t}{t}=\infty
$$

then

$$
m^{*}(r, u)>\cos \pi \rho\{1-h(r)\} M(r, u)
$$

on a sequence of $r \rightarrow \infty$.

If

$$
\int^{\infty} h(t) \frac{d t}{t}<\infty
$$

there is a subharmonic function of order $\rho(0<\rho<1)$ and mean type for which

$$
m^{*}(r, u)<\{\cos \pi \rho-h(r)\} M(r, u) \quad\left(r \geqq r_{0}\right) .
$$

Baernstein [1] generalized Theorem A as follows.

THEOREM C. Let $u(z)$ be a nonconstant subharmonic function in $\boldsymbol{C}$. Let $\beta$ and $\lambda$ be numbers with $0<\lambda<\infty, 0<\beta \leqq \pi, \beta \lambda<\pi$. Then either (a) there exist arbitrarily large values of $r$ for which the set of $\theta$ such that $u\left(r e^{i \theta}\right)>\cos \beta \lambda$. $M(r, u)$ contains an interval of length at least $2 \beta$, or else $(b) \lim _{r \rightarrow \infty} r^{-\lambda} M(r, u)$ exists, and is positive or $\infty$.

For $\beta=\pi$, this is Theorem A.

In this note we shall prove the following result.

THEOREM. Let $u(z)$ be subharmonic of order $\rho(0<\rho<\infty)$ and mean type in C. Let $\beta$ be a number satısfying $0<\beta \leqq \pi$ and $\beta \rho<\pi / 2$. Suppose that $h(r)$ is positive and continuous for $r \geqq r_{0}$ and, for each $s>0$,

$$
\frac{h(s r)}{h(r)} \longrightarrow 1 \quad(r \rightarrow \infty) \text {. }
$$

Further assume that $h(r) \rightarrow 0(r \rightarrow \infty), h^{\prime}(r)>-O\left(r^{-1}\right)(r \rightarrow \infty)$ and

$$
\int^{\infty} h(t) \frac{d t}{t}=\infty \text {. }
$$

Then there exist arbitrarily large values of $r$ for which the set of $\theta$ such that $u\left(r e^{i \theta}\right)>\cos \beta \rho\{1-h(r)\} M(r, u)$ contains an interval of length at least $2 \beta$.

For $\beta=\pi$, this is the first half of Theorem $B$.

2. Proof of Theorem. Since we are interested in results valid for large values of $r$, we may assume without loss of generality that $u(z)$ is harmonic in a neighborhood of $z=0$. Let $\beta$ be a number satisfying $0<\beta \leqq \pi$. Put

$$
u(r, \beta, \phi)=\int_{-\beta}^{+\beta} u\left(r e^{\imath\left(\omega+\xi^{\prime}\right)}\right) d \omega \quad(r>0, \phi: \text { real }) .
$$


For $r e^{\imath \beta}$ fixed, $u(\gamma, \beta, \phi)$ is a continuous (periodic) function of $\phi$ (cf. [4, Lemma 3]). Therefore each fixed $r e^{2 \beta}$, there exists a $\phi_{0}\left(-\pi \leqq \phi_{0}<\pi\right)$ satisfying

$$
N(r, \beta, u) \equiv \sup _{\rho} u(r, \beta, \phi)=u\left(r, \beta, \phi_{0}\right) .
$$

Here we set

$$
\mu(r, u)=\inf \left\{u\left(r e^{\imath \omega}\right) ; \omega \in\left[\phi_{0}-\beta, \phi_{0}+\beta\right]\right\} .
$$

In order to prove our theorem, it is sufficient to show that

$$
\mu(r, u)>\cos \beta \rho\{1-h(r)\} M(r, u)
$$

for a sequence $r=r_{n} \rightarrow \infty$.

If $v(z)=u(z)-u(0)$, then

$$
\mu(r, u)=\mu(r, v)+u(0), \quad M(r, u)=M(r, v)+u(0) .
$$

By Theorem $\mathrm{C}$, we may assume that

$$
\lim _{r \rightarrow \infty} r^{-\rho} M(r, u)=\alpha \quad(\alpha \text {; a positive constant }) .
$$

Now assume that our assertion is proved for $v(z)$, that is,

$$
\mu(r, v)>\cos \beta \rho\{1-(h(r) / 2)\} M(r, v)
$$

for a sequence of $r=r_{n} \rightarrow \infty$. Then for $r=r_{n}$

$$
\begin{aligned}
\mu(r, u) & >\cos \beta \rho\{1-(h(r) / 2)\} M(r, u)+u(0)[1-\cos \beta \rho\{1-(h(r) / 2)\}] \\
& >\cos \beta \rho\{1-(h(r) / 2)\} M(r, u)-|u(0)| \quad\left(n \geqq n_{0}\right) \\
& >\cos \beta \rho\left\{1-(h(r) / 2)-O\left(r^{-\rho}\right)\right\} M(r, u) \\
& >\cos \beta \rho\{1-h(r)\} M(r, u) \quad\left(n \geqq n_{1}\right),
\end{aligned}
$$

since $h(r)$ is slowly varying. Thus we may assume that $u(0)=0$.

Set

$$
B(t)=N\left(t^{r}, \beta, u\right),
$$

where $\gamma=\beta / \pi$. Since $u(z)$ is of order $\rho$ and mean type, we have

$$
B(t) \leqq 2 \beta M\left(t^{\gamma}, u\right)=O\left(t^{\gamma \rho}\right) \quad(t \rightarrow \infty) .
$$

Since $\gamma \rho=\beta \rho / \pi<1 / 2$, the Poisson integral

$$
b\left(r e^{i \theta}\right)=\frac{1}{\pi} \int_{0}^{\infty} B(t) \frac{r \sin \theta}{t^{2}+r^{2}+2 t r \cos \theta} d t
$$

is harmonic in the slit plane $|\arg z|<\pi$, is zero on the positive axis and tends to $B(r)$ as $\theta \rightarrow \pi-$. By Proposition 1 in [1], $B(t)$ is a nondecreasing convex function of $\log t(0<t<\infty)$. Differentiating (4) with respect to $\theta$, we have 


$$
\begin{aligned}
& b_{\theta}\left(r e^{i \theta}\right)=\frac{1}{\pi} \int_{0}^{\infty} \log \left|1+\frac{r e^{2 \theta}}{t}\right| d B_{1}(t) \quad(|\theta|<\pi), \\
& b_{\theta}(-r) \equiv \lim _{\theta \rightarrow \pi_{-}} \frac{B(r)-b\left(r e^{i \theta}\right)}{\pi-\theta}=\lim _{\theta \rightarrow \pi_{-}} b_{\theta}\left(r e^{i \theta}\right)=\frac{1}{\pi} \int_{0}^{\infty} \log \left|1-\frac{r}{t}\right| d B_{1}(t),
\end{aligned}
$$

(6) where $B_{1}(t)$ denotes the logarithmic derivative of $B(t)$. Since $B(t)$ is a nondecreas-
ing convex function of $\log t, B_{1}(t)$ exists a. e., and is a nonnegative nondecreasing function of $t$. It follows from (5) and (6) that $b_{\theta}(z)$ is subharmonic in $C$ and that

$$
m^{*}\left(r, b_{\theta}\right)=b_{\theta}(-r), \quad M\left(r, b_{\theta}\right)=b_{\theta}(r) .
$$

Using (3) and the fact that $B(0)=0$, we easily see

$$
\begin{aligned}
& B_{1}(t)=O\left(t^{\gamma \rho}\right) \quad(t \rightarrow \infty), \\
& \lim _{t \rightarrow 0}\left(\log \frac{1}{t}\right) B_{1}(t)=0 .
\end{aligned}
$$

Hence

(8)

$$
\begin{aligned}
b_{\theta}(r) & =\frac{1}{\pi} \int_{0}^{\infty} \log \left(1+\frac{r}{t}\right) d B_{1}(t) \\
& =\frac{1}{\pi}\left[\log \left(1+\frac{r}{t}\right) B_{1}(t)\right]_{0}^{\infty}-\frac{1}{\pi} \int_{0}^{\infty} \frac{-\frac{r}{t^{2}}}{1+\frac{r}{t}} B_{1}(t) d t \\
& =\frac{r}{\pi} \int_{0}^{\infty} \frac{1}{t+r} \frac{B_{1}(t)}{t} d t=\frac{r}{\pi} \int_{0}^{\infty} \frac{d B(t)}{t+r} \\
& =\frac{r}{\pi}\left[\frac{B(t)}{t+r}\right]_{0}^{\infty}-\frac{r}{\pi} \int_{0}^{\infty} \frac{-B(t)}{(t+r)^{2}} d t \\
& =\frac{r}{\pi} \int_{0}^{\infty} \frac{B(t)}{(t+r)^{2}} d t=O\left(r^{r \rho}\right) \quad(r \rightarrow \infty) .
\end{aligned}
$$

In view of (7) and (8), we have

$$
M\left(r, b_{\theta}\right)=O\left(r^{r \rho}\right) \quad(r \rightarrow \infty) .
$$

Define $D$ by $D=\{z ; 0<\arg z<\beta\}$. Let $H(z)$ be the harmonic function in $D$ defined by $H(z)=b\left(z^{1 / r}\right)$. Taking the estimate (3) into consideration, Baernstein's reasoning in [1, pp 192-195] gives

$$
\left\{\begin{array}{l}
H_{\theta}(r) \geqq 2 M(r, u) \\
H_{\theta}(r)+H_{\theta}\left(r e^{\imath \beta}\right) \leqq 2[\mu(r, u)+M(r, u)] \quad(0<r<\infty) .
\end{array}\right.
$$

It follows from (10) and (7) that if $H_{\theta}(r)+H_{\theta}\left(r e^{\imath \beta}\right) \geqq 0$ then 


$$
\frac{\mu(r, u)}{M(r, u)} \geqq \frac{H_{\theta}\left(r e^{\imath \beta}\right)}{H_{\theta}(r)}=\frac{b_{\theta}\left(-r^{1 / r}\right)}{b_{\theta}\left(r^{1 / r}\right)}=\frac{m^{*}\left(r^{1 / \gamma}, b_{\theta}\right)}{M\left(r^{1 / \gamma}, b_{\theta}\right)} .
$$

By (9) the order of $b_{\theta}(z)$ is less than or equal to $\gamma \rho(<1 / 2)$. Assume first that $b_{\theta}(z)$ has order less than $\gamma \rho$. The classical $\cos \pi \rho$ theorem gives the estimate

$$
\frac{m^{*}\left(r, b_{\theta}\right)}{M\left(r, b_{\theta}\right)}>\cos \pi(\gamma \rho)=\cos \beta \rho \quad\left(r=r_{n} \rightarrow \infty\right) .
$$

Combining (11) and (12), we have

$$
\frac{\mu(r, u)}{M(r, u)}>\cos \beta \rho \quad\left(r=r_{n}^{r} \rightarrow \infty\right) .
$$

Assume next that $b_{\theta}(z)$ is of order $\gamma \rho$ and minimal type. In this case, we use Theorem A to obtain the estimate (12), so that (13) follows.

It remains to consider the case that $b_{\theta}(z)$ is of order $\gamma \rho$ and mean type. Define $h_{1}(t)$ by $h_{1}(t)=h\left(t^{r}\right)$. Then $h_{1}(t)$ is positive and continuous for $t \geqq r_{0}^{1 / r}$, and for each $s>0$,

$$
\frac{h_{1}(s t)}{h_{1}(t)}=\frac{h\left(s^{\gamma} t^{r}\right)}{h\left(t^{\gamma}\right)} \longrightarrow 1 \quad(t \rightarrow \infty)
$$

Further $h_{1}(t) \rightarrow 0(t \rightarrow \infty), h_{1}^{\prime}(t)=\gamma t^{\gamma-1} h^{\prime}\left(t^{\gamma}\right)>-O\left(t^{-1}\right)(t \rightarrow \infty)$, and

$$
\int_{r_{0}^{1 / \gamma}}^{\infty} h_{1}(t) \frac{d t}{t}=\int_{\gamma_{0}^{1 / \gamma}}^{\infty} h\left(t^{r}\right) \frac{d t}{t}=\frac{1}{\gamma} \int_{\gamma_{0}}^{\infty} h(t) \frac{d t}{t}=\infty .
$$

Hence by Theorem $\mathrm{B}$, the inequality

$$
\frac{m^{*}\left(r, b_{\theta}\right)}{M\left(r, b_{\theta}\right)}>\cos \pi(\gamma \rho)\left\{1-h_{1}(r)\right\}=\cos \beta \rho\left\{1-h_{1}(r)\right\}
$$

holds on a sequence $r=r_{n} \rightarrow \infty$. Combining (14) and (11), we have

$$
\frac{\mu(r, u)}{M(r, u)}>\cos \beta \rho\{1-h(r)\} \quad\left(r=r_{n}^{r} \rightarrow \infty\right) .
$$

This completes the proof of our theorem.

\section{REFERENCES}

[1] A. Baernstein II, A generalization of $\cos \pi \rho$ theorem, Trans. Amer. Math. Soc. Vol. 193 (1974), 181-197.

[2] P.D. BARRY, On the growth of entire functions, Mathematical essays dedicated to A. J. Macintyre, Ohio Univ. Press (1970), 43-60.

[3] B. KJellberG, A theorem on the minimum modulus of entire functions, Math. Scand. 12 (1963), 5-11.

[4] H. Ueda, An extremal problem for subharmonic functions of $\mu_{*}<1 / 2$, Kodai Math. J. Vol. 4, No. 3 (1981), 457-479.

Department of Mathematics

Daido Institute of Technology

Daido-cho, Minami-Ku, Nagoya, Japan 